\title{
Lamivudine: fading into the mists of time
}

\author{
Jonggi Choi and Young-Suk Lim \\ Department of Gastroenterology, Liver Center, Asan Medical Center, University of Ulsan College of Medicine, Seoul, Korea
}

Keywords: Lamivudine; Entecavir; Nucleos(t)ide analogues

\section{See Article on Page 331}

The treatment goal for patients with chronic hepatitis B (CHB) is reducing liver related mortalities from cirrhosis and hepatocellular carcinoma (HCC). For the past 20 years, single or combination regimen of nucleos(t)ide analogues (NUCs) has been widely used for this purpose and partly achieved the treatment goal in CHB patients. Hepatitis B e antigen (HBeAg)-negative CHB has been regarded as challenging to treat owing to the rapid and aggressive progression of the disease. ' Since the phase 3 study by Lai et al. ${ }^{2}$ entecavir has been widely used in treatment-naïve HBeAg-negative CHB patients and showed its higher efficacy than lamivudine in the real world data. ${ }^{3}$ International guidelines also recommend entecavir as the preferred choice for first-line agent for CHB patients along with tenofovir disoproxil fumarate, rather than lamivudine. ${ }^{4-6}$

Although the higher efficacy of entecavir compared with lamivudine has reported in previous randomized trial, ${ }^{2}$ Lee et al. conducted a randomized controlled trial to compare the 'long-term' efficacy and safety during 5 years in treatment-naive $\mathrm{HBeAg-negative}$ Korean CHB patients whose genotype are mostly genotype C? In the current issue of Clinical and Molecular Hepatology, this study showed long-term entecavir treatment was superior to lamivudine as quite well expected. Virologic response, defined according to the AASLD guidelines, ${ }^{4}$ was achieved in $95.0 \%$ of entecavir-treated patients and $47.6 \%$ of lamivudine-treated patients $(P<0.0001)$ after 240 weeks of treatment. Notably, $26(42.6 \%)$ of lamivudinetreated patients occurred virologic breakthrough until week 96 , whereas only one patient of entecavir-treated patient developed virologic breakthrough. This patient with virologic breakthrough at week 48 did not have resistance to entecavir and was found to have undetectable HBV DNA at week 60 and 192. The demerit of Lee's study is the small sample size and high drop-out rate as the authors mentioned in discussion. According to the study flow depicted in figure 1, most common cause of drop-out was treatment failure/lack of efficacy $(n=24)$ and belonged to lamivudine-treated group. However, these high drop-outs might be expected in consequence of the lower efficacy and high virologic breakthrough in lamivudine-treated group.

Regarding the safety and tolerability, Lee's study reported both entecavir and lamivudine were well tolerated with a low incidence of adverse events which were not related to the study drugs through 5 year of study period. This accumulates supporting

\section{Abbreviations:}

CHB; chronic hepatitis B, HCC; hepatocellular carcinoma, NUCs; nucleos(t)ide analogues

\section{Corresponding author : Young-Suk Lim}

Department of Gastroenterology, Asan Medical Center, University of Ulsan College of Medicine, 88 Olympic-ro 43-gil, Songpa-gu, Seoul 05505, Korea Tel: +82-2-3010-5933, Fax: +82-2-485-5782

E-mail:limys@amc.seoul.kr

http://orcid.org/0000-0002-1544-577X 
evidence for long-term use of entecavir in terms of safety concern considering the need of almost indefinite treatment with NUC until more potent antiviral agent appears.

We previously conducted a large historical cohort study with 5,374 CHB patients (1785 HBeAg-negative patients: $33.2 \%){ }^{8}$ In this study, entecavir showed a low risk of death or transplantation than lamivudine, which fulfills in part the treatment goal in CHB patients, albeit the development of HCC did not differ between two groups. ${ }^{8}$ Based on previous reports including our large historical cohort study and Lee's randomized study, lamivudine should not be considered any more as an option for NUCs in treatmentnaïve CHB patients. Next research questions we might have is whether the long-term outcomes differ or not between entecavir and tenofovir, which are equivalent in terms of antiviral potency in the setting of treatment naive CHB patients.

Lamivudine, which is the first NUC for CHB patients, showed its efficacy in preventing liver disease progression but was also responsible for emerging drug-resistant mutants causing more severe hepatitis flares, disease progression, and death due to the low genetic barrier to resistance. ${ }^{9}$ No international guidelines recommend its use as a first-line option in CHB patients any more. Lamivudine, this old star is fading into the mists of time.

\section{Authors' contribution}

All authors contributed to drafting of the manuscript and approved the final version for submission.

\section{Acknowledgement}

This paper was supported by grants from the Korean National Health Clinical Research (NHCR) project, Ministry of Health \& Welfare, Republic of Korea (HC15C3380).

\section{Conflicts of Interest}

The authors have no conflicts to disclose.

\section{REFERENCES}

1. Hadziyannis SJ, Papatheodoridis GV. Hepatitis B e antigen-negative chronic hepatitis B: natural history and treatment. Semin Liver Dis 2006;26:130-141.

2. Lai CL, Shouval D, Lok AS, Chang TT, Cheinquer H, Goodman Z, et al. Entecavir versus lamivudine for patients with $\mathrm{HBeAg}$-negative chronic hepatitis B. N Engl J Med 2006;354:1011-1120.

3. Seto WK, Lam YF, Fung J, Wong DK, Huang FY, Hung IF, et al. Changes of HBsAg and HBV DNA levels in Chinese chronic hepatitis B patients after 5 years of entecavir treatment. J Gastroenterol Hepatol 2014;29:1028-1034.

4. Terrault NA, Bzowej NH, Chang KM, Hwang JP, Jonas MM, Murad $\mathrm{MH}$, et al. AASLD guidelines for treatment of chronic hepatitis $\mathrm{B}$. Hepatology 2016;63:261-283.

5. Sarin SK, Kumar M, Lau GK, Abbas Z, Chan HLY, Chen CJ, et al. Asian-Pacific clinical practice guidelines on the management of hepatitis B: a 2015 update. Hepatol Int 2016;10:1-98.

6. European Association for the Study of the Liver. EASL 2017 Clinical Practice Guidelines on the management of hepatitis B virus infection. J Hepatol 2017;67:370-398

7. Lee KS, Kweon YO, Um SH, Kim BH, Lim YS, Paik SW, et al. Efficacy and safety of entecavir versus lamivudine over 5 years of treatment: a randomized controlled trial in Korean patients with hepatitis B e antigen-negative chronic hepatitis B. Clin Mol Hepatol 2017;23:331339.

8. Lim YS, Han S, Heo NY, Shim JH, Lee HC, Suh DJ. Mortality, liver transplantation, and hepatocellular carcinoma among patients with chronic hepatitis B treated with entecavir vs lamivudine. Gastroenterology 2014;147:152-161.

9. Papatheodoridis GV, Dimou E, Dimakopoulos K, Manolakopoulos $S$, Rapti I, Kitis $G$, et al. Outcome of hepatitis B e antigen-negative chronic hepatitis B on long-term nucleos(t)ide analog therapy starting with lamivudine. Hepatology 2005;42:121-129. 